\title{
Spectroscopic characterization of a sample of southern visual binaries $^{\star}, \star \star$
}

\author{
S. Desidera ${ }^{1}$, R. G. Gratton ${ }^{1}$, S. Lucatello ${ }^{1}$, R. U. Claudi ${ }^{1}$, and T. H. Dall ${ }^{2}$ \\ 1 INAF - Osservatorio Astronomico di Padova, Vicolo dell' Osservatorio 5, 35122 Padova, Italy \\ e-mail: desidera@pd.astro.it \\ 2 European Southern Observatory, Casilla 19001, Santiago, Chile
}

Received 23 January 2006 / Accepted 22 February 2006

\section{ABSTRACT}

\begin{abstract}
Aims. We present the spectroscopic characterization of 56 pairs of visual binaries with similar components, based on high resolution spectra acquired with FEROS at ESO La Silla.

Methods. For all stars, we measured radial and rotational velocities and CaII H\&K emission.

Results. Five previously unknown double lined spectroscopic binaries were found. Six other pairs show velocity differences that are not compatible with the orbital motion of the wide pair, indicating the presence of further companion(s) in the system. The fraction of visual binaries that contain additional spectroscopic components is $27 \pm 10 \%$, compatible with other literature estimates. The ages of the components of the pairs derived from chromospheric activity typically show apparent differences of about 0.2 dex. A few pairs show a rather large difference in activity level, but in most cases this is consistent with the variability of chromospheric emission observed for the Sun along its magnetic cycle.
\end{abstract}

Key words. stars: binaries: visual - stars: binaries: spectroscopic - stars: activity - stars: rotation - techniques: radial velocities techniques: spectroscopic

\section{Introduction}

Visual binaries represent a class of binaries for which individual components can be studied in detail. The large separation makes the effects of tidal and magnetic interactions negligible, so that the components can be considered as evolving in isolation. Characterization of the individual components allows the dispersion of several physical parameters in coeval systems to be evaluated. In binary systems with evolved components, accurate isochrone ages can also be derived.

Differential chemical abundance analysis of main sequence stars with temperature differences lower than $\sim 300 \mathrm{~K}$ may reach sensitivities to abundance differences between the components down to 0.02 dex (Desidera et al. 2004a). This allows us to place very tight limits on the amount of planetary material accreted during the main-sequence lifetime. Differences in the lithium content between the components of binary systems, observed in a significant fraction of the pairs (Martin et al. 2002), may shed light on the mechanism(s) that alter the abundance of this element during the evolution a star.

The measurement of rotational velocities and chromospheric activity are also important. Differences between the components in these quantities are useful for determining the dispersion of these parameters at a fixed age. The availability of activity indicators may help in the selection of stars suitable for planets searches. Slowly-rotating, chromospherically quiet stars are the best targets for radial velocity surveys, because the radial

^ Based on observations collected at the European Southern Observatory, Chile, using FEROS spectrograph (proposal

ID: 69.D-0338-70.D-0081).

$\star \star$ Tables 2-4 are only available in electronic form at http://www.edpsciences.org velocity jitter caused by stellar activity is lower (e.g. Saar et al. 1998). On the other hand, nearby young stars are the best targets for direct imaging searches, because of the larger intrinsic luminosity of brown dwarfs and planets at young ages (Burrows et al. 1997).

The multiplicity of the components of visual binaries is also relevant. In fact, the number of components in multiple systems may provide crucial clues to the star formation mechanism (Tokovinin 2004; Goodwin \& Kroupa 2005). Statistical studies indicate that the distribution of orbital and stellar parameters in close binary systems with another distant third component may be different from those of double stars (Tokovinin $\&$ Smekhov 2002). Furthermore, peculiar dynamical configurations and orbital characteristics were found in high-multiplicity systems. Measurement of the radial velocity of the components might reveal additional companions. In the case of visual binaries, the presence of companions can be inferred in many cases from single-epoch spectra of the components, when the velocity difference between the components exceed what is expected on the basis of the long-period orbit of the wide binary (about $2-3 \mathrm{~km} \mathrm{~s}^{-1}$ for systems with projected separation of a few hundredths $\mathrm{AU}$, such as those studied in this paper). Identifying close spectroscopic binaries among the components of the wide pairs allows us also to clean the sample from systems for which mutual interaction might not be negligible.

With the main goals of $i$ ) increasing the sample of visual binaries with high precision differential abundance measurement and ii) of characterizing targets in visual binaries for future planet search programs, we acquired high resolution spectra of a sample of southern wide binaries.

The pairs were selected from the Hipparcos Multiple Star Catalog (Double and Multiples: Component solutions; 
ESA 1997) adopting the following criteria: $V<10.0$, parallax greater than 10 mas, magnitude difference lower tham $1.0 \mathrm{mag}^{1}$, colors in the range $0.45<(B-V)_{\mathrm{T}}<1.1$ or spectral type later than F5, and a projected separation larger than 4 arcsec (to avoid contamination of the spectra). The projected physical separation spans the range between 28 and $5000 \mathrm{AU}$.

The similarity between the components is important to obtain differential abundance measurements with errors smaller than 0.02 dex. The chemical composition analysis of the suitable pairs is presented in the companion paper, Desidera et al. (2006b). In this paper, we present our measurements of radial velocities, Ca II H\&K emission and rotational velocities for the 56 pairs we observed.

Thirty-one pairs have independent proper motion in the Hipparcos Multiple Star Catalog. Seven of them show a difference at $\geq 3 \sigma$ level in at least one of the coordinates. The consistency of RV and other stellar properties (activity level and rotational velocity studied here, metallicity from Desidera et al. 2006b) suggests that the proper motion difference is not real. In some cases, it is very likely due to the orbital motion of the wide pair. Hereafter we assume that all the pairs are physical with the possible exception of the composite system HIP 40831.

The outline of the paper is as follows: the observations and data reduction are described in Sect. 2; the radial velocities are presented in Sect. 3; the spectroscopic binaries in the sample are discussed in Sect. 4; Sects. 5 and 6 present the measurements of the projected rotational velocity and the chromospheric activity; in Sect. 7 we discuss individual systems worthy of special mention; we summarize the results in Sect. 8 .

\section{Observations and data reduction}

High resolution spectra of 56 pairs were acquired using the FEROS spectrograph at ESO in two observing runs (August 2002 and March 2003). The spectrograph was attached to the $1.5 \mathrm{~m}$ and $2.2 \mathrm{~m}$ telescopes for the first and second runs respectively. The spectral format includes the spectral region 3900-9200 $\AA$, at a nominal resolving power of $R=48000$.

Due to some technical problem and the smaller telescope aperture during the first run, it was not possible to observe all the originally selected pairs. Some standard stars with known chromospheric emission were also observed in order to provide a suitable calibration.

The on-line data reduction procedure was not fully adequate for our main scientific goal. In fact, the difference in equivalent widths of multiple spectra for the same star showed systematic trends with wavelength. Furthermore, the spectra obtained with the simultaneous thorium calibration (August 2002 run) show some contamination by the lamp lines.

A new reduction was then performed, using $\mathrm{IRAF}^{2}$. Extraction of apertures was performed by tracing one of the two peaks due to the image slicer and by assigning an asymmetric size to the aperture to include most of the light from the target. Analysis of the FWHM of thorium lines indicates that our reduction procedure does not degradate spectral resolution.

Inter-order background was subtracted, and flat-fielding division performed using the flat field spectrum extracted on the

\footnotetext{
1 Three pairs with larger magnitude difference, namely HIP 70264, HIP 76446, and HIP 78024, were also observed and included in this paper.

${ }_{2}^{2}$ IRAF is distributed by the National Optical Observatory, which is operated by the Association of Universities for Research in Astronomy, Inc., under contract with the National Science Foundation.
}

Table 1. Properties of double-lined spectroscopic binaries detected in our spectra: identification, radial velocities, $F W H M$ of spectral lines corrected for instrumental broadening, and ratio of equivalent width at about $6400 \AA$. The epoch of the observations is listed in Table 4.

\begin{tabular}{cccc}
\hline \hline Object & $\begin{array}{c}R V \\
\mathrm{~km} \mathrm{~s}^{-1}\end{array}$ & $\begin{array}{c}F W H M \\
\mathrm{~km} \mathrm{~s}^{-1}\end{array}$ & $E W$ ratio \\
\hline HIP 35564Ba & $7.22 \pm 0.52$ & $31.2 \pm 5.5$ & $0.22 \pm 0.03$ \\
HIP 35564Bb & $76.02 \pm 0.27$ & $16.9 \pm 2.6$ & \\
& & & \\
HIP 35733Aa & $72.34 \pm 0.19$ & $12.45 \pm 0.45$ & $0.82 \pm 0.04$ \\
HIP 35733Ab & $-4.97 \pm 0.24$ & $12.13 \pm 0.74$ & \\
HIP 76816Aa & $-48.83 \pm 0.37$ & $22.6 \pm 1.2$ & $0.33 \pm 0.01$ \\
HIP 76816Ab & $-29.9 \pm 1.2$ & $18.5 \pm 1.2$ & \\
& & & \\
HIP 78416Aa & $58.9 \pm 1.0$ & $44.2 \pm 2.1$ & $0.87 \pm 0.06$ \\
HIP 78416Ab & $-1.8 \pm 2.2$ & $41.1 \pm 4.0$ & \\
& & & \\
HIP 81395A & $-24.97 \pm 0.31$ & $20.6 \pm 1.2$ & $0.76 \pm 0.06$ \\
HIP 81395B & $-3.10 \pm 0.51$ & $15.9 \pm 1.2$ & \\
\hline
\end{tabular}

same pixels as the science spectra, efficiently removing interference fringes in the red part of the spectra and the blaze function pattern.

For the spectra of the August 2002 run an additional step was performed at the beginning of the procedure: an image with no stellar flux, but with thorium lines in the second channel, was used to remove the residual contamination of the Th lines, scaling the flux with respect to each science frame. The procedure succesfully removes the contamination of Th lines on science spectra. A more severe clipping factor was used in determining the background level, to minimize the effect of any impefection on the substraction of thorium lines.

\section{Radial velocity}

Radial velocities (hereafter $R V$ ) of program stars were derived by means of the cross correlation technique using the FXCOR task in IRAF. Comparison of 10 stars with radial velocity measured by Nidever et al. (2002) shows a mean difference $R V_{\mathrm{us}}-R V_{\mathrm{N} 02}=-0.015 \pm 0.029 \mathrm{~km} \mathrm{~s}^{-1}$, with a dispersion of $0.087 \mathrm{~km} \mathrm{~s}^{-1}$. This confirms the reliability of the radial velocities measured with the FEROS spectrograph.

Individual radial velocities are listed in Table 4. Errors are estimated from the scatter of the $R V$ of individual spectral orders, adding a $0.1 \mathrm{~km} \mathrm{~s}^{-1}$ systematic error in quadrature.

\section{Spectroscopic binaries}

Several components of the visual binaries turned out to be itself spectroscopic binaries. We describe here their identification from our data and the literature and we discuss their frequency in our sample.

\subsection{Double-line spectroscopic binaries}

Five stars in our sample are resolved as double-lined spectroscopic binaries (hereafter SB2) in our spectra. Table 1 lists the $R V$ of the components, the $F W H M$ of the spectral lines corrected for instrumental broadening, and the ratio of equivalent width measured on a few isolated lines in the range 6000-6700. .

Two further SB2 are HIP 43692A = HD 76037A (Desidera et al. 2006a) and HIP 45734B (Covino et al. 1997). HIP 40831B 


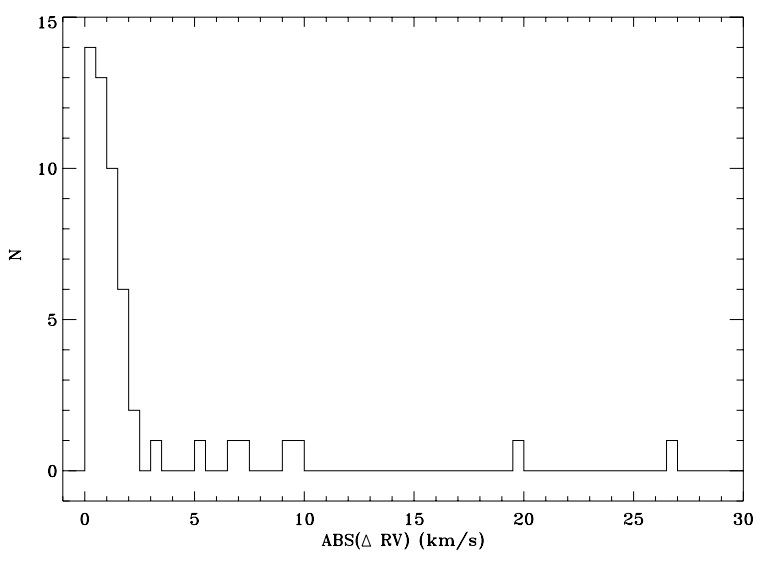

Fig. 1. Distribution of the absolute value of the $R V$ difference between the components of the wide binaries studied here. Most of them have $R V$ difference below $2-3 \mathrm{~km} \mathrm{~s}^{-1}$, compatible with the expected binary motion and observational errors. The pairs with $\Delta R V>5 \mathrm{~km} \mathrm{~s}^{-1}$ should likely host an additional spectroscopic components. A few stars are outside the plot limits.

may also be a SB2 system. Its spectrum appears to be composite, with the spectral features of a solar-type star dominating most of the spectrum, but with the strong Hydrogen lines typical of a late A star in the blue. The physical association between these two stars is not clear and is discussed in Sect. 7.

\subsection{Suspected spectroscopic binaries}

For the wide binaries we are considering, radial velocity difference between the components caused by the orbital motion should be less than $2-3 \mathrm{~km} \mathrm{~s}^{-1}$. When the velocity differences are larger than this limit, most likely one (or both) components of the visual pair is a spectroscopic binary.

Six pairs show $R V$ difference larger than $5 \mathrm{~km} \mathrm{~s}^{-1}$ : HIP 25434/6, HIP 51578, HIP 61465/6, HIP 79819, HIP 100045, HIP 110832. As the faintness of the secondary makes its spectral lines not visible in the spectrum, it is not possible to identify which of the components hosts a further companion by using a single spectrum.

An alternative explanation to the observed RV difference is that some of these pairs might only be optical. Only two of the six pairs listed above have independent proper motion determinations in the Hipparcos Multiple Star Catalog. Those for HIP 61465/6 agree within errors, while those for HIP 25434-HIP 25436 are different at about a $2 \sigma$ level. However, the small RV difference $\left(7 \mathrm{~km} \mathrm{~s}^{-1}\right)$, the Hipparcos parallaxes that agree within the (rather large) errors, and the fast rotational velocity observed for both stars (suggesting commong young age) make a chance association unlikely. More likely, the binarity of one of the components induces the relative astrometric motion detected by Hipparcos.

Two further spectroscopic binaries are HIP $76603=$ HD 139461 (Tokovinin \& Gorynya 2001) and HIP 64030A (Desidera et al. 2006c).

\subsection{Statistic of multiplicity}

From the results of Sects. 4.1 and 4.2 we can derive the frequency of systems with at least three components in our sample. These are 15 or 16 out of 56 pairs $(27-29 \pm 10 \%$, respectively), depending on the inclusion of HIP 40831 or not. This fraction is compatible with the results by Tokovinin \& Smekhov (2002) and
Tokovinin (2004), about $33 \%$ of visual binaries contain additional spectroscopic components. Some observational incompleteness is certainly present, since single-epoch observations are not sensitive to spectroscopic binaries with an amplitude lower than about 2-3 $\mathrm{km} \mathrm{s}^{-1}$ and a pair can by chance have a small radial velocity difference at the epoch of our observation even if one of the components is itself a large-amplitude spectroscopic binary. On the other hand, some biases may favor the inclusion of triples in our sample. The Malmquist bias (magnitude limit was one of the selection criteria) favors the inclusion of stars with companions significantly contributing to the integrated light. This might explain the relatively large number of SB2 systems. Another bias is due to the fact that parallax errors are on average larger for pairs with additional unrecognized components (see e.g. Söderhjelm 1999), because the binary motion is not taken into account in the parallax solution. This makes the inclusion of high multiplicity systems within the nominal $100 \mathrm{pc}$ limit more likely. A detailed statistical evaluation of the frequency of high multiplicity systems in our sample is beyond the scope of this paper.

\section{Rotational velocity}

The rotational velocity was derived by means of a suitable calibration of the $F W H M$ of stellar lines. The $F W H M$ of each spectral line was determined as part of the equivalent width measurement for the abundance analysis using a Gaussian fitting. More than 300 lines were measured for slowly rotating stars. A relation of $F W H M$ vs. equivalent width was then derived (see. Bragaglia et al. 2001). The adopted $F W H M$ is the result of the fitting relation for an equivalent width of $50 \mathrm{~m} \AA$. This technique is applicable to stars with rotational velocities lower than about $40 \mathrm{~km} \mathrm{~s}^{-1}$. For the remaining stars, $v \sin i$ was estimated directly from the line profile of a few isolated lines in the red portion of the spectra.

The other broadening mechanisms (macroturbulence, microturbulence, thermal broadening, instrumental broadening) have to be properly taken into account when deriving the rotational velocity. Such broadening represents a lower envelope of the $F W H M$ as a function of $(B-V)^{3}$ (see e.g. Valenti \& Fischer 2005). It is then quadratically subtracted to the observed $F W H M$, obtaining the broadening caused by stellar rotation. The latter is finally calibrated using stars with known $v \sin i$ (Table 2, available only in electronic form), giving half weight to the Nordstrom et al. (2004) results:

$v \sin i=0.008+0.658 \times F W H M_{\mathrm{rot}}-0.002 \times F W H M_{\mathrm{rot}}^{2}$.

The resulting dispersion of the calibration is about $1.8 \mathrm{~km} \mathrm{~s}^{-1}$ considering all the calibration stars and $1.2 \mathrm{~km} \mathrm{~s}^{-1}$ escluding those by Nordstrom et al. (2004, Fig. 3).

For the few evolved stars, the instrinsic broadening is larger than the adopted one, derived mostly from main sequence stars. The rotational velocity is then overestimated for these objects.

\section{Chromospheric emission}

The inclusion of the $\mathrm{H}$ and $\mathrm{K} \mathrm{CaII} \mathrm{lines} \mathrm{in} \mathrm{the} \mathrm{spectral} \mathrm{format} \mathrm{al-}$ lowed us to measure the chromospheric emission of our program star and to derive the age from its strength.

\footnotetext{
${ }^{3} B-V$ is available for most of the program stars from Tycho. Tycho photometry has been calibrated into the standard system as in Bessell (2000).
} 


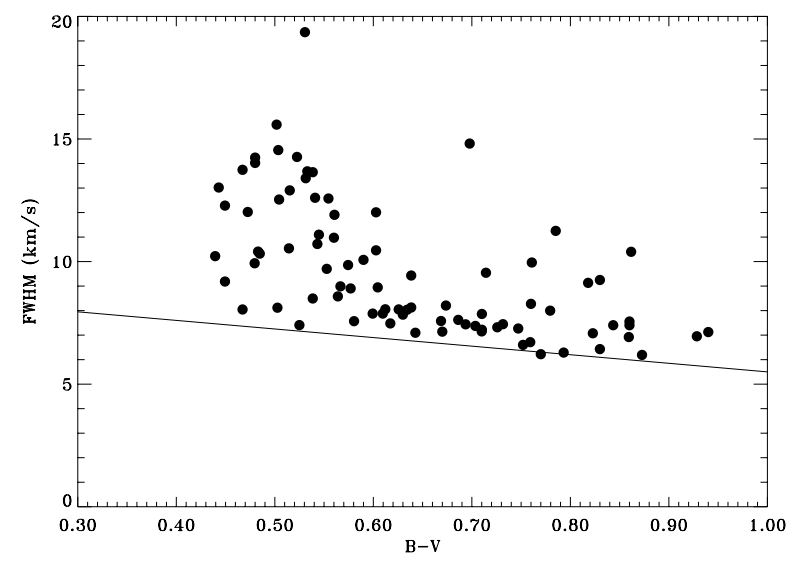

Fig. 2. $F W H M$ (corrected for instrumental broadening) vs. $B-V$ for program stars. The lower envelope shown as a continuous line is taken as our estimate of the instrinsic broadening due to other causes than rotation (thermal, macro, and microturbulence). The stars with the largest rotational velocities are outside of the plot limits.

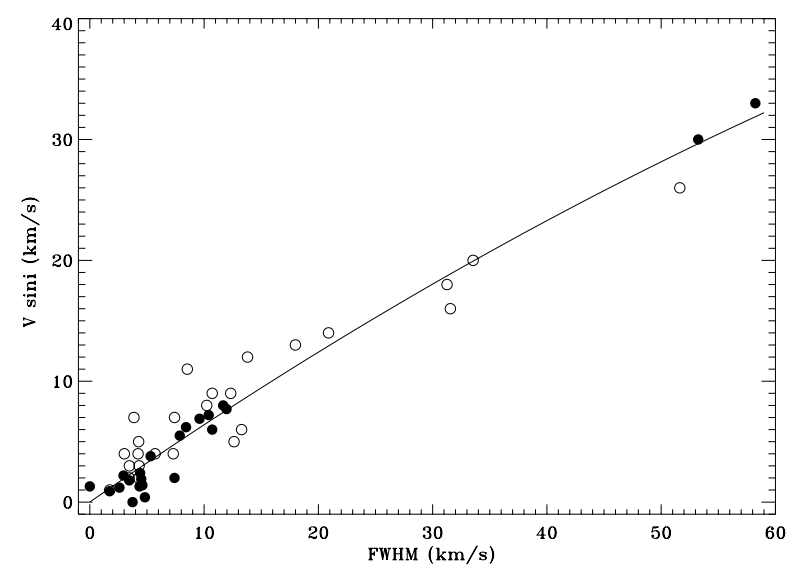

Fig. 3. Calibration of $F W H M$ (corrected for broadening mechanisms other than rotation) and $v \sin i$ from the literature. Empty circles are the stars in common with Nordstrom et al. (2004), and filled circles are the stars from other literature sources (see Table 2). The adopted calibration is overplotted as a solid line.

\subsection{Measurement and calibration}

We determined the chromospheric activity index $S$ by measuring the fluxes in the $1 \AA$ wide band centered on $\mathrm{H}$ and $\mathrm{K} \mathrm{Ca}$ II lines and in two adjacent continuum windows that are $20 \AA$ wide. For homogeneity with the published literature, the resulting $S$ index has to be calibrated into the Mount Wilson system. To this aim, a number of standard stars with known activity level were observed during our second run. Furthermore, some of our program stars also have a published CaII H\&K determination (Table 3, available only in electronic form).

The calibration of our instrumental $S$ index $\left(S_{\text {FEROS }}\right)$ into the standard system is (Fig. 4):

$S_{\mathrm{MW}}=0.027+1.029 \times S_{\text {FEROS }}$

with rms dispersion of 0.018 .

Photon noise errors of the measured $S$ index are always below 0.005 and in most cases below 0.002 . However, as is typical of S-index measurement from high resolution spectra, errors are probably dominated by systematics, due to e.g. imperfect corrections for scattered light and blaze function and to problems with order extraction in a region with low flux (Wright et al. 2004).

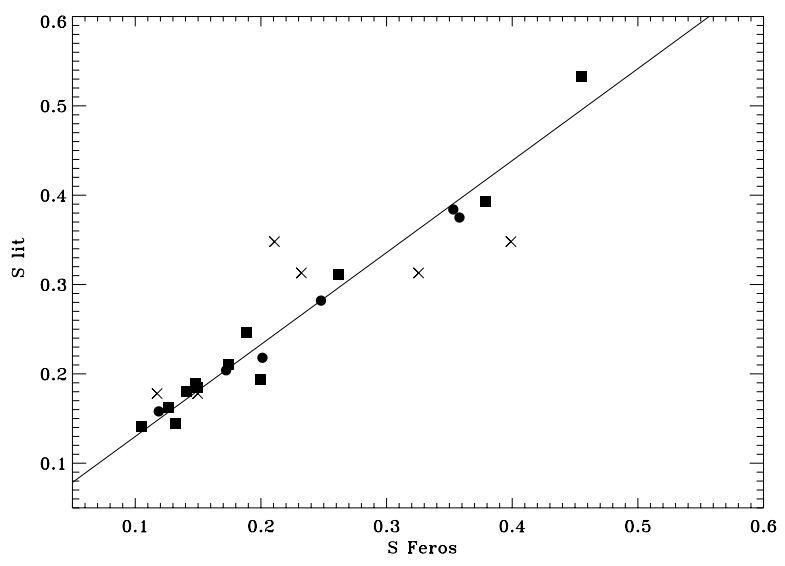

Fig. 4. Calibration of instrumental $S$ index measured on FEROS spectra in the standard Mount Wilson system. Filled circles and squares represent stars observed in the first and second observing runs. The crosses represent the pairs for which only the $S$-index measurement composite of the two components are available in the literature. They were not used in the derivation of the calibration.

Such errors are difficult to quantify but from the difference in the $S$ index measured on consecutive spectra and, in a few cases, on spectra taken in two consecutive nights we estimate that the typical measurement error of the $S$ index is about 0.01. The intrinsic variability of chromospheric emission should then contribute significantly to the scatter of the calibration.

The calibration of Noyes et al. (1984) was used to derive the chromospheric flux in the CaII H \& $\mathrm{K}$ lines $\log R^{\prime}(\mathrm{HK})$ from the measured $S$ index

$R_{\mathrm{HK}}^{\prime}=R_{\mathrm{HK}}-R_{\text {phot }}$

where $R_{\text {phot }}$ is the photospheric component approximated as

$\log R_{\text {phot }}=-4.898+1.918(B-V)^{2}-2.893(B-V)^{3}$

and $R_{\mathrm{HK}}$ is the total CaII H\&K flux (chromospheric+photospheric) given as

$R_{\mathrm{HK}}=1.340 \times 10^{-4} S C_{\mathrm{cf}}$

where

$\log C_{\mathrm{cf}}=1.13(B-V)^{3}-3.91(B-V)^{2}+2.84(B-V)-0.47$. (6)

The typical absolute errors on $\log R^{\prime}(\mathrm{HK})$ caused by the uncertainty in the $S$ index (0.01) and $B-V$ color (about 0.03 mag, because of the low quality of Tycho photometry for our stars) is about 0.1 dex.

The values of chromospheric activity derived for the components of HIP 114914 = HD 219542 supersede those reported in Desidera et al. (2003), which are based on a preliminary calibration performed on the data reduced with the on-line pipeline and used as calibrators objects observed during the instrument commissioning (Kaufer et al. 1999).

\subsection{Ages from chromospheric activity}

Ages were derived from the observed $\log R^{\prime}(\mathrm{HK})$ using the calibration by Donahue (1993). The validity of this calibration has been recently questioned for very young stars (Song et al. 2004), as well as for old stars (Pace \& Pasquini 2004).

We are observing pairs of stars supposed to be coeval with a separation large enough to make negligible the magnetic activity enhancements due to proximity effects (save for the few 


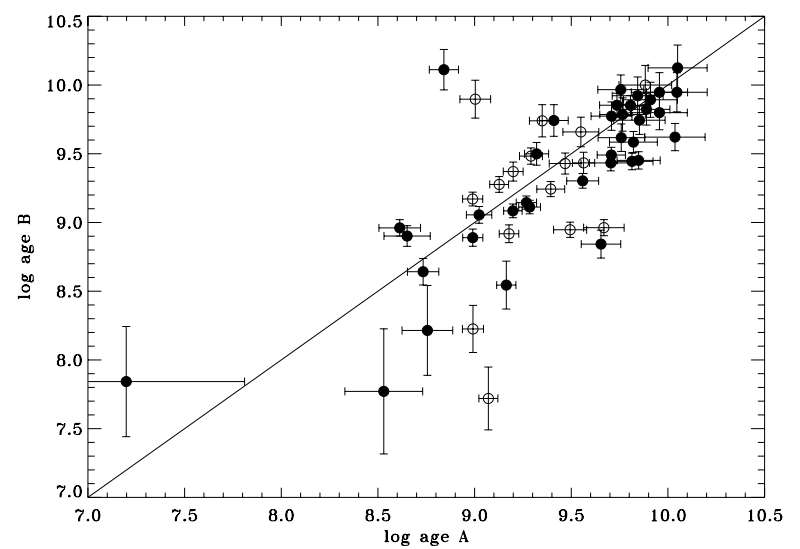

Fig. 5. Ages of the components of the wide binaries studied in this paper derived from our measurement of chromospheric activity. Empty circles: pairs for which the choromosphric activity measurement is less reliable (pairs with a SB2 component; rotational velocity larger than $40 \mathrm{~km} \mathrm{~s}^{-1}$; magnitude difference larger than $1 \mathrm{mag}, B-V$ color outside the validity of the calibration by Noyes et al. (1984). Filled circles: the other pairs.

pairs with additional components, whose orbits have to be determined). We can then address the issue of the reliability of relative ages based on chromospheric activity, by comparing the ages derived for the two components (Fig. 5).

In some cases, a spurious difference in the age derived from chromospheric activity might be the result of several factors. Pairs with SB2 components might have disturbed line profiles, and the flux ratio at CaII $\mathrm{H} \& \mathrm{~K}$ wavelength should be different from the one used for the $B-V$ color $^{4}$. Stars with $v \sin i>$ $40 \mathrm{~km} \mathrm{~s}^{-1}$ have wide enough spectral lines so that $\mathrm{H} \& \mathrm{~K}$ core emission in not entirely included in the adopted $1 \AA$ band (see Sect. 6.1). Furthermore, there are stars in our sample with colors that are outside the range on which the Noyes et al. (1984) calibration is defined $(0.44<B-V<0.9)$, and the age calibration should also not be applicable to the pair whose primary is a giant (HIP 70264). At young ages the very steep age dependence in the adopted calibration implies large errors in the derived ages even when the activity level of the components is consistent within errors (HIP 37923/18, HIP 44817/14, HIP 51266).

Excluding all these cases, the typical difference in the ages measured from the activity level results of about 0.2 dex, not much larger than the error bars of the activity measurement itself $(0.15$ dex $)$. However, in a few cases, the differences in the activity level between the components are significant and cause apparent age differences between the components above 0.25 dex. For comparison, the age of the Sun derived from the activity level at the minimum and maximum of a solar cycle differs by 0.56 dex (Henry et al. 1996).

From Fig. 5 it also appears that the apparent age difference between the components, as determined from chromospheric activity, is larger for stars 2-5 Gyr old than at very old ages. This might be explained by a decline in the variability of magnetic activity between solar age and very old ages. A few cases with rather large apparent age difference probably due to a real difference in the activity level between the components are discussed in Sect. 7.

${ }^{4}$ In the case of HIP 78416A, the chromospheric emission is clearly shifted in velocity with respect to the center of the absorption line. It appears that the component $\mathrm{Ab}$ is more active than Aa. The $S$ index measurement in Table 4 is the one measured for the $R V$ of Aa.

\section{Comments on individual systems}

We comment here on individual systems that deserve mention for their binarity (Sects. 4.1, 4.2) or different activity level (Sect. 6).

- HIP 35564 is already known as a quadruple system (Saar et al. 1990). The secondary HIP $35564 \mathrm{~B}=$ HD 57583 is itself a triple system. HD 57583A is orbited by the very tight binary HD $57583 \mathrm{BaBb}$. The lines of HD $57583 \mathrm{~A}$ and $\mathrm{Ba}$ are visible in Saar et al. and our spectra. The orbital period of the A-B pair is 122 days. Nordstrom et al. (2004) report a single-epoch measurement of the $R V$ of HIP $35564 \mathrm{~A}=$ HD $57852\left(17.3 \pm 1.5 \mathrm{~km} \mathrm{~s}^{-1}\right)$ that is significantly different from our $(26.85 \pm 0.79)$. This difference suggests that HIP 35564 A may itself be a spectroscopic binary, making the system a quintuple.

- HIP 35733 is flagged as an SB2 by Nordstrom et al. (2004), without providing further details. In our spectrum the lines are resolved well, with a velocity difference of about $77 \mathrm{~km} \mathrm{~s}^{-1}$.

- HIP 40831B is a possible SB2 system. Its spectrum appears to be composite, with the spectral features of a solar type star dominating most of the spectrum, but with the strong Hydrogen lines typical of a late A star in the blue. Its companion HIP 40831A is classified as G0V (Houk 1982), and its position in the color-magnitude diagram adopting the Hipparcos parallax $\left(M_{V}=4.44 \pm 0.27, B-V=0.54 \pm\right.$ 0.03 ) is consistent with this classification. The origin of the A-type features is then not clear, since a normal A main sequence star would overcome the brightness of the two solar-type stars if placed at the same distance, and the system would have an absolute magnitude that is not compatible with those derived from Hipparcos. The small $R V$ differences between the components of the wide pair makes it unlikely that HIP 40831B is a short period binary. The proper motion of the components of HIP 40831 listed in the Hipparcos Multiple Star Catalog are different, with those of HIP 40831B showing huge errors $\left(\mu_{\alpha}(\mathrm{A})=-81.16 \pm 1.12\right.$, $\mu_{\delta}(\mathrm{A})=60.55 \pm 1.20, \mu_{\alpha}(\mathrm{B})=-18.22 \pm 20.91, \mu_{\delta}(\mathrm{B})=$ $10.12 \pm 17.02)$. This indicates problems in the Hipparcos solution, probably due to the unrecognized presence of the A star. The possibility that the A type component is an unrelated background star seems viable, but further studies are required to reveal the nature of this object.

- HIP 43692A = HD 76037A is another double-lined spectroscopic binary. A small asymmetry of the line profiles in our FEROS spectrum suggests the presence of a companion. The reality of this companion is confirmed by high resolution spectra acquired by us with SARG at TNG (Desidera et al. 2006a).

- HIP 45734B is a known SB2 already (Covino et al. 1997) not visible as a binary in our spectrum. According to Covino et al. (1997), HIP $45734 \mathrm{~B}$ is in the T Tauri phase. In our spectrum it shows a very large $S$ index, which implies a chromospheric emission outside the range of validity of the age calibration. The star shows also a prominent $\mathrm{H}_{\alpha}$ emission. The primary instead has a much lower emission.

- HIP 61465/6 = HD109556: no core Ca II H\&K emission is clearly seen for both components. It is possible that the difference in the age measured from chromospheric activity is due to uncertainly in the background level for the low $\mathrm{S} / \mathrm{N}$ spectrum of the secondary.

- HIP 70386 = HD 126246: the primary shows both a larger chromospheric emission and larger projected rotational 
velocity. This suggests that the primary is really more active and rotating faster than the secondary. This enhanced activity of the primary is not due to a close companion, since even $1 M_{\mathrm{J}}$ planets in close orbits are excluded by the high-precision radial velocity monitoring going on at SARG (Desidera et al. 2006a).

- HIP 76816A, HIP 78416A, and HIP 81395 are all SB2 not previously identified as binaries.

- HIP 78024 = HD 142661: a very different activity level is apparent from the spectra. The large magnitude difference may in principle introduce some spurious effect (cf. Paulson et al. 2002) but it is unlikely that this can explain the whole difference.

- HIP 100045: the components show an $R V$ difference $\left(-6.75 \mathrm{~km} \mathrm{~s}^{-1}\right)$ indicating binarity for one of the components. The comparison with the $R V$ reported by Nordstrom et al. (2004) allows us to identify the binary component (the primary).

- HIP 110832 = HD 212746: the very large difference in the activity level between the components may be partially due to the low $\mathrm{S} / \mathrm{N}$ of the spectrum of the primary. However, the primary also has a faster rotational velocity. The $R V$ difference indicates that one of the components is a spectroscopic binary. A companion in orbit close enough to tidally spin up the primary would explain the observed differences, but of course this scenario needs confirmation. The possibility of chance projection should also be considered. No independent proper motion determination for the two components are listed in the Hipparcos Multiple Star Catalog. However, data from the Washington Double Star Catalog (Mason et al. 2001) show only small changes in the position angle and projected separation over more than one century $\left(\rho_{1879}=6.7^{\prime \prime}, \theta_{1879}=186^{\circ}, \rho_{1998}=6.4^{\prime \prime}, \theta_{1998}=183^{\circ}\right)$, indicating physical association.

- HIP 114914 = HD 219542: the large difference in the activity level between the components of this binary has been already noted by us (Desidera et al. 2004b) in our discussion of the origin of the low-amplitude radial velocity variations of HD 219542B.

- HIP 20552 = HD28255, HIP 58813/15 = HD104760/59, and HIP $103438=$ HD 199065: intrinsic differences between the components in the strength of chromospheric emission are evident from the spectra.

\section{Conclusion}

We have performed a spectroscopic characterization of the components of 56 visual binaries with similar components. Visual inspection of the spectra and the measurement of radial velocities allowed identification of some new double-lined spectroscopic binaries and several single-lined spectroscopic binary candidates. The fraction of spectroscopic components in our sample agrees within errors with estimates from the literature.

The measurements of projected rotational velocity and of Ca II chromospheric emission allowed us to further characterize the stars. A few stars show rather large differences in their activity level, but this appears consistent with the variability actually observed for the Sun during its magnetic activity cycle. The typical apparent difference in ages derived from chromospheric activity results about 0.2 dex.
Some pairs with low rotational velocity and low magnetic activity level are well suited for the search for planets using radial velocity and for a detailed differential chemical abundance analysis, for which sensitivities down to a few earth masses of accreted rocky material can be achieved. This work is presented in a companion paper (Desidera et al. 2006b).

Active, probably young, stars are instead interesting as possible targets for next generation, direct imaging instruments such as the VLT Planet Finder (Beuzit et al. 2005). Some potential candidates were identified and will be studied in more detail in the future.

Acknowledgements. This research has made use of the SIMBAD database, operated at the CDS, Strasbourg, France. We warmly thank Ivo Saviane for useful discussions. This work was partially funded by COFIN 2004 "From stars to planets: accretion, disk evolution and planet formation" by the Ministero Università e Ricerca Scientifica Italy. We thank the referee, Dr. A. Tokovinin, for a prompt and thoughtful report.

\section{References}

Baliunas, S. L., Donahue, R. A., Soon, W. H., et al. 1995, ApJ, 438, 269 Benz, W., \& Mayor, M. 1984, A\&A, 138, 183 Bessell, M. S. 2000, PASP, 112, 961

Beuzit, J.-L., et al. 2005, Direct Imaging of Exoplanets: Science and Techniques, Proc. IAU Coll., 200, in press

Bragaglia, A., Carretta, E., Gratton, R., et al. 2001, AJ, 121, 327

Burrows, A., Marley, M., Hubbard, W. B., et al. 1997, ApJ, 491, 856

Covino, E., Alcala, J. M., Allain, S., et al. 1997, A\&A, 328, 187

Desidera, S., Gratton, R. G., Endl, M., et al. 2003, A\&A, 405, 207

Desidera, S., Gratton, R. G., Scuderi, S., et al. 2004a, A\&A, 420, 683

Desidera, S., Gratton, R. G., Endl, M., et al. 2004b, A\&A, 420, L27

Desidera, S., Gratton, R. G., Claudi, R. U., et al. 2006a, Proceedings of ESO Workshop Multiple Stars Across the HR Diagram, in press

Desidera, S., Gratton, R. G., Lucatello, S., \& Claudi, R. U. 2006b, A\&A, in press Desidera, S., et al. 2006c, A\&A, in preparation

Donahue, R. A. 1993, Ph.D. Thesis, New Mexico State University

ESA 1997, The Hipparcos and Tycho Catalogues, ESA SP-1200

Fuhrmann, K. 2004, Astron. Nachr., 325, 1

Goodwin, S. P., \& Kroupa, P. 2005, A\&A, 439, 565

Henry, T. J., Soderblom, D. R., Donhaue, R. A., \& Baliunas, S. L. 1996, AJ, 111, 439

Houk, N. 1982, Michigan Spectral Survey, Ann Arbor, Dep. Astron., Univ. Michigan, 3

Kaufer, A., Stahl, O., Tubbesing, S., et al. 1999, ESO Messenger, 95, 8

Martin, E. L., Basri, G., Pavlenko, Y., \& Lyubchik, Y. 2002, ApJ, 579, 437

Mason, B. D., Wycoff, G. L., Hartkopf, W. I., Douglass, G. G., \& Worley, C. E. 2001, AJ, 122, 3466

Nidever, D. L., Marcy, G. W., Butler, R. P., Fischer, D. A., \& Vogt, S. S. 2002 , ApJS, 141, 503

Nordstrom, B., Mayor, M., Andersen, J., et al. 2004, A\&A, 418, 989

Noyes, R. W., Hartmann, L. W., Baliunas, S. L., Duncan, D. K., \& Vaughan, A. H. 1984, ApJ, 279, 763

Pace, G., \& Pasquini, L. 2004, A\&A, 426, 1021

Paulson, D. B., Saar, S. H., Cochran, W. D., \& Hatzes, A. 2002, AJ, 124, 572

Reiners, A., \& Schmitt, J. H. M. M. 2003, A\&A, 398, 647

Saar, S. H., Nordstrom, B., \& Andersen, J. 1990, A\&A, 235, 291

Saar, S. H., Butler, R. P., \& Marcy, G. W. 1998, ApJ, 498, L153

Söderhjelm, S. 1999, A\&A, 341, 121

Song, I., Zuckerman, B., \& Bessell, M. S. 2004, ApJ, 614, L125

Tinney, C. G., McCarthy, C., Jones, H. R., et al. 2002, MNRAS, 332, 759

Tokovinin, A. A. 2004, Rev. Mex. Astron. Astrofis., 21, 7

Tokovinin, A. A., \& Gorynya, N. A. 2001, A\&A, 374, 227

Tokovinin, A. A., \& Smekhov, M. G. 2002, A\&A, 382, 118

Valenti, J., \& Fischer, D. A. 2005, ApJS, 159, 141

Wright, J. T., Marcy, G. W., Butler, R. P., \& Vogt, S. S. 2004, ApJS, 152, 261

Zuckerman, B., \& Webb, R. A. 2000, ApJ, 535, 959 


\section{Online Material}


S. Desidera et al.: Spectroscopic characterization of a sample of southern visual binaries, Online Material p 2

Table 2. Calibration of $v \sin i$.

\begin{tabular}{|c|c|c|c|}
\hline Object & $v \sin i_{\text {lit }}$ & $F W H M_{\text {rot }}$ & Reference \\
\hline HIP 4540 A & 18.0 & 31.26 & 1 \\
\hline HIP 10175A & 0.4 & 4.82 & 2 \\
\hline HIP 10175B & 0.0 & 3.74 & 2 \\
\hline HIP 20610 & 26.0 & 51.63 & 1 \\
\hline HIP 20612 & 16.0 & 31.55 & 1 \\
\hline HIP 35564B & 20.0 & 33.54 & 1 \\
\hline HIP 40831A & 11.0 & 8.53 & 1 \\
\hline HIP 43692A & 5.0 & 12.62 & 1 \\
\hline HIP 43692B & 12.0 & 13.80 & 1 \\
\hline HIP 44817A & 5.0 & 4.27 & 1 \\
\hline HIP 44817B & 3.0 & 3.46 & 1 \\
\hline HIP 45734A & 6.0 & 13.28 & 1 \\
\hline HIP 49520A & 4.0 & 3.02 & 1 \\
\hline HIP 55288A & 8.0 & 10.23 & 1 \\
\hline HIP 56280A & 8.0 & 11.67 & 3 \\
\hline HIP 56280B & 9.0 & 12.33 & 1 \\
\hline HIP 58813 & 3.0 & 4.31 & 1 \\
\hline HIP 58815 & 2.0 & 3.44 & 1 \\
\hline HIP 62596A & 7.2 & 10.40 & 2 \\
\hline HIP 62596B & 6.9 & 9.60 & 2 \\
\hline HIP 65352 & 1.3 & 0.00 & 2 \\
\hline HIP 65355 & 0.9 & 1.73 & 2 \\
\hline HIP 70386B & 4.0 & 5.71 & 1 \\
\hline HIP 73674A & 4.0 & 4.22 & 1 \\
\hline HIP 73674B & 7.0 & 3.86 & 1 \\
\hline HIP 74432A & 1.8 & 3.45 & 2 \\
\hline HIP 74432B & 1.0 & 1.74 & 1 \\
\hline HIP 76602 & 2.0 & 7.41 & 4 \\
\hline HIP 76603 & 6.0 & 10.71 & 4 \\
\hline HIP 76602 & 7.0 & 7.41 & 1 \\
\hline HIP 76603 & 9.0 & 10.71 & 1 \\
\hline HIP 84405A & 1.4 & 4.60 & 5 \\
\hline HIP 84405B & 1.3 & 4.35 & 5 \\
\hline HIP 99803A & 33.0 & 58.24 & 6 \\
\hline HIP 99803B & 30.0 & 53.24 & 6 \\
\hline HIP 100045A & 13.0 & 17.99 & 1 \\
\hline HIP $100045 B$ & 4.0 & 7.30 & 1 \\
\hline HIP 103438A & 5.5 & 7.87 & 6 \\
\hline HIP 114167A & 14.0 & 20.89 & 1 \\
\hline HIP 114914A & 1.9 & 4.50 & 7 \\
\hline HIP 114914B & 2.4 & 4.40 & 7 \\
\hline HD 38392 & 2.0 & 4.26 & 1 \\
\hline HD 38393B & 7.7 & 11.96 & 3 \\
\hline HD 45067 & 6.2 & 8.42 & 2 \\
\hline HD 76151 & 1.2 & 2.59 & 2 \\
\hline HD 115617 & 2.2 & 2.95 & 2,3 \\
\hline HD 152391 & 3.8 & 5.32 & 2 \\
\hline
\end{tabular}

References: 1. Nordstrom et al. (2004); 2. Valenti \& Fischer (2005); 3. Reiners \& Schmitt (2003); 4. Fuhrmann (2004); 5. Benz \& Mayor (1984); 6. Zuckermann \& Webb (2000); 7. Desidera et al. (2003).
Table 3. Calibration of $S$ index on the Mount Wilson system.

\begin{tabular}{ccccl}
\hline \hline Object & $S_{\text {FEROS }}$ & $S_{\text {lit }}$ & Run & Remarks \\
\hline HIP 10175A & 0.2012 & 0.218 & 1 & 1 \\
HIP 10175B & 0.2478 & 0.282 & 1 & 1 \\
HIP 20552 & 0.1479 & 0.189 & 2 & 2 \\
HIP 45734 & 0.2617 & 0.311 & 2 & 3 \\
HIP 62596A & 0.1741 & 0.211 & 2 & 1 \\
HIP 62596B & 0.1495 & 0.185 & 2 & 1 \\
HIP 65176A & 0.1174 & $0.178^{a}$ & 2 & 3 \\
HIP 65176B & 0.1497 & $0.178^{a}$ & 2 & 3 \\
HIP 65352A & 0.1406 & 0.180 & 2 & 1 \\
HIP 65352B & 0.1998 & 0.194 & 2 & 1 \\
HIP 74432 & 0.1321 & 0.145 & 2 & 1 \\
HIP 78416A & 0.2322 & $0.313^{a}$ & 1 & 3 \\
HIP 78416B & 0.3255 & $0.313^{a}$ & 1 & 3 \\
HIP 84405A & 0.3580 & 0.375 & 1 & 4 \\
HIP 84405B & 0.3531 & 0.384 & 1 & 4 \\
HIP 103438A & 0.2108 & $0.348^{a}$ & 1 & 3 \\
HIP 103438B & 0.3990 & $0.348^{a}$ & 1 & 3 \\
HIP 114914A & 0.1187 & 0.158 & 1 & 1 \\
HIP 114914B & 0.1723 & 0.204 & 1 & 1 \\
& & & & \\
HD 38392 & 0.4551 & 0.533 & 2 & 1 \\
HD 45067 & 0.1048 & 0.141 & 2 & 4 \\
HD 76151 & 0.1883 & 0.246 & 2 & 4 \\
HD 115617 & 0.1268 & 0.162 & 2 & 1,4 \\
HD 152391 & 0.3786 & 0.393 & 2 & 1,4
\end{tabular}

References: 1. Wright et al. (2004); 2. Tinney et al. (2002); 3. Henry et al. (1996); 4. Baliunas et al. (1995). ${ }^{a} \mathrm{~A}+\mathrm{B}$. 
S. Desidera et al.: Spectroscopic characterization of a sample of southern visual binaries, Online Material $p 3$

Table 4. Results of our measurement for program stars: HIP and HD names, identification of the component (A, B) date of the observations, radial velocity, $B-V$ color, $F W H M$ of spectral lines (corrected for instrumental broadening), projected rotational velocity, $S$ index calibrated on the Mount Wilson scale, chromospheric emission flux, logarithm of age derived from chromospheric activity, and additional remarks. For stars with $v \sin i>40 \mathrm{~km} \mathrm{~s}^{-1}$, only lower limits to chromospheric emission were measured.

\begin{tabular}{|c|c|c|c|c|c|c|c|c|c|c|c|}
\hline $\begin{array}{l}\text { Name } \\
\text { HIP }\end{array}$ & $\begin{array}{l}\text { Name } \\
\text { HD }\end{array}$ & Comp. & $\begin{array}{c}\text { JD } \\
-2450000\end{array}$ & $\begin{array}{c}R V \\
\mathrm{~km} \mathrm{~s}^{-1}\end{array}$ & $B-V$ & $\begin{array}{l}F W H M \\
\mathrm{~km} \mathrm{~s}^{-1}\end{array}$ & $\begin{array}{c}v \sin i \\
\mathrm{~km} \mathrm{~s}^{-1}\end{array}$ & $S$ & $\log R_{\mathrm{HK}}^{\prime}$ & $\begin{array}{c}\log \text { age } \\
y r\end{array}$ & Remarks \\
\hline 3290 & 4001 & $\mathrm{~A}$ & 2498.350 & $-2.73 \pm 0.12$ & 0.47 & 12.02 & 6.1 & 0.13 & -5.21 & 10.05 & \\
\hline 3290 & & B & 2498.401 & $-0.81 \pm 0.13$ & 0.51 & 10.54 & 5.0 & 0.14 & -5.14 & 9.95 & \\
\hline 4540 & 5659 & A & 2500.306 & $0.60 \pm 0.36$ & 0.47 & 31.26 & 18.2 & 0.22 & -4.65 & 9.20 & \\
\hline 4540 & & B & 2500.326 & $1.83 \pm 0.20$ & 0.43 & 39.63 & 22.7 & 0.19 & -4.76 & 9.37 & \\
\hline 10175 & 13357 & A & 2500.352 & $25.20 \pm 0.11$ & 0.67 & 8.21 & 3.1 & 0.23 & -4.70 & 9.27 & \\
\hline 10175 & & $\mathrm{~B}$ & 2500.393 & $26.01 \pm 0.11$ & 0.73 & 7.45 & 2.4 & 0.28 & -4.61 & 9.14 & \\
\hline 20552 & 28255 & A & 2720.978 & $-4.61 \pm 0.10$ & 0.63 & 7.83 & 2.5 & 0.18 & -4.88 & 9.56 & \\
\hline 20552 & & $\mathrm{~B}$ & 2720.986 & $-3.15 \pm 0.11$ & 0.70 & 7.38 & 2.2 & 0.23 & -4.72 & 9.30 & \\
\hline 20610 & 29058 & A & 2720.994 & $-0.97 \pm 0.21$ & 0.47 & 53.63 & 29.6 & 0.31 & -4.43 & 8.73 & \\
\hline 20612 & & $\mathrm{~B}$ & 2721.003 & $0.20 \pm 0.14$ & 0.50 & 31.55 & 18.4 & 0.32 & -4.41 & 8.64 & \\
\hline 25436 & 35996 & A & 2721.014 & $32.17 \pm 1.30$ & 0.41 & & 112 & $>0.25$ & $>-4.57$ & $<9.07$ & SB1? \\
\hline 25434 & 274197 & $\mathrm{~B}$ & 2721.026 & $24.94 \pm 0.33$ & 0.51 & & 40 & 0.39 & -4.30 & 7.72 & SB1? \\
\hline 28852 & 41683 & A & 2721.043 & $-4.74 \pm 0.21$ & 0.41 & 43.77 & 24.8 & 0.18 & -4.82 & 9.47 & \\
\hline 28852 & & $\mathrm{~B}$ & 2721.055 & $-5.13 \pm 0.17$ & 0.47 & 31.80 & 18.5 & 0.19 & -4.80 & 9.43 & \\
\hline 35564 & 57852 & A & 2721.993 & $26.85 \pm 0.79$ & 0.42 & & 68 & 0.23 & -4.64 & 9.18 & SB1? \\
\hline 35564 & 57853 & $\mathrm{~B}$ & 2721.997 & $0.32 \pm 0.18$ & 0.58 & 33.54 & 19.5 & 0.29 & -4.49 & 8.92 & SB2 \\
\hline 35733 & 58038 & A & 2722.004 & $72.11 \pm 0.22$ & 0.54 & & & 0.24 & -4.60 & 9.13 & SB2 \\
\hline 35733 & & B & 2722.011 & $28.50 \pm 0.10$ & 0.57 & 8.99 & 3.6 & 0.22 & -4.70 & 9.28 & \\
\hline 36817 & 60584 & $\mathrm{~A}$ & 2721.069 & $-4.13 \pm 0.38$ & 0.44 & & 41 & $>0.19$ & $>-4.78$ & $<9.39$ & \\
\hline 36817 & 60585 & $\mathrm{~B}$ & 2721.073 & $-4.92 \pm 0.36$ & 0.46 & & 41 & $>0.21$ & $>-4.68$ & $<9.24$ & \\
\hline 37923 & 63608 & $\mathrm{~A}$ & 2721.078 & $17.65 \pm 0.11$ & 0.79 & 11.25 & 6.0 & 0.59 & -4.27 & 7.20 & \\
\hline 37918 & 63581 & $\mathrm{~B}$ & 2721.102 & $17.70 \pm 0.11$ & 0.78 & 8.00 & 3.2 & 0.53 & -4.31 & 7.84 & \\
\hline 39409 & 66491 & $\mathrm{~A}$ & 2719.997 & $47.74 \pm 0.10$ & 0.75 & 7.27 & 2.3 & 0.27 & -4.65 & 9.20 & \\
\hline 39409 & & $\mathrm{~B}$ & 2720.020 & $48.98 \pm 0.11$ & 0.67 & 7.58 & 2.4 & 0.28 & -4.58 & 9.08 & \\
\hline 40831 & 70326 & A & 2722.021 & $31.24 \pm 0.11$ & 0.54 & 11.10 & 5.5 & 0.16 & -4.95 & 9.67 & \\
\hline 40831 & & $\mathrm{~B}$ & 2722.038 & $30.34 \pm 0.12$ & 0.57 & 9.86 & 4.5 & 0.28 & -4.51 & 8.96 & composite \\
\hline 43692 & 76037 & A & 2720.045 & $1.26 \pm 0.13$ & 0.50 & 14.55 & 8.0 & 0.14 & -5.14 & 9.96 & SB2 \\
\hline 43692 & & B & 2720.072 & $2.54 \pm 0.12$ & 0.50 & 15.59 & 8.7 & 0.15 & -5.03 & 9.80 & \\
\hline 44817 & 78977 & $\mathrm{~A}$ & 2720.159 & $6.11 \pm 0.11$ & 0.84 & 7.40 & 2.8 & 0.47 & -4.44 & 8.76 & \\
\hline 44814 & 78964 & $\mathrm{~B}$ & 2720.194 & $6.08 \pm 0.12$ & 0.86 & 6.92 & 2.3 & 0.59 & -4.34 & 8.21 & \\
\hline 45734 & 81485 & A & 2720.230 & $5.13 \pm 0.11$ & 0.70 & 14.81 & 8.4 & 0.30 & -4.56 & 9.06 & \\
\hline 45734 & & B & 2720.243 & $-39.43 \pm 1.56$ & 0.83 & & & 1.01 & -4.06 & & SB2 \\
\hline 49520 & 87743 & A & 2720.102 & $-0.36 \pm 0.11$ & 0.62 & 7.48 & 2.0 & 0.22 & -4.71 & 9.28 & \\
\hline 49520 & & B & 2720.086 & $0.34 \pm 0.11$ & 0.60 & 7.88 & 2.5 & 0.26 & -4.59 & 9.11 & \\
\hline 51266 & 90885 & $\mathrm{~A}$ & 2721.118 & $14.49 \pm 0.11$ & 0.83 & 9.25 & 4.5 & 0.50 & -4.39 & 8.53 & \\
\hline 51266 & & $\mathrm{~B}$ & 2721.131 & $16.17 \pm 0.11$ & 0.82 & 9.13 & 4.4 & 0.58 & -4.30 & 7.77 & \\
\hline 51578 & 91561 & A & 2722.064 & $32.63 \pm 0.25$ & 0.42 & 44.24 & 25.1 & 0.18 & -4.84 & 9.49 & SB1? \\
\hline 51578 & & $\mathrm{~B}$ & 2722.089 & $-4.72 \pm 0.62$ & 0.47 & 62.16 & 33.4 & 0.27 & -4.50 & 8.95 & SB1? \\
\hline 55288 & & A & 2722.114 & $19.80 \pm 0.12$ & 0.50 & 12.53 & 6.5 & 0.15 & -5.01 & 9.76 & \\
\hline 55288 & & B & 2722.122 & $19.12 \pm 0.10$ & 0.71 & 7.22 & 2.0 & 0.14 & -5.15 & 9.97 & \\
\hline 56280 & 100287 & A & 2721.203 & $5.72 \pm 0.12$ & 0.53 & 13.68 & 7.4 & 0.15 & -5.01 & 9.77 & \\
\hline 56280 & 100286 & $\mathrm{~B}$ & 2721.207 & $7.63 \pm 0.12$ & 0.52 & 14.27 & 7.8 & 0.15 & -5.02 & 9.79 & \\
\hline 58298 & 103817 & $\mathrm{~A}$ & 2721.243 & $-3.85 \pm 0.12$ & 0.47 & 8.05 & 2.1 & 0.14 & -5.14 & 9.96 & \\
\hline 58298 & & $\mathrm{~B}$ & 2721.257 & $-3.88 \pm 0.12$ & 0.50 & 8.12 & 2.4 & 0.14 & -5.14 & 9.95 & \\
\hline 58813 & 104760 & A & 2721.214 & $6.29 \pm 0.11$ & 0.63 & 8.03 & 2.8 & 0.15 & -5.04 & 9.81 & \\
\hline 58815 & 104759 & B & 2721.230 & $6.10 \pm 0.11$ & 0.73 & 7.32 & 2.2 & 0.21 & -4.81 & 9.44 & \\
\hline 58864 & 104830 & $\mathrm{~A}$ & 2722.131 & $-40.58 \pm 0.12$ & 0.45 & 9.19 & 3.5 & 0.14 & -5.07 & 9.85 & \\
\hline 58862 & 104829 & $\mathrm{~B}$ & 2722.160 & $-40.61 \pm 0.11$ & 0.63 & 8.05 & 2.8 & 0.16 & -5.00 & 9.74 & \\
\hline 59743 & 106515 & $\mathrm{~A}$ & 2720.119 & $20.66 \pm 0.11$ & 0.79 & 6.29 & 0.6 & 0.16 & -5.04 & 9.81 & \\
\hline 59743 & & $\mathrm{~B}$ & 2720.132 & $19.94 \pm 0.11$ & 0.83 & 6.43 & 1.3 & 0.16 & -5.07 & 9.85 & \\
\hline 61466 & 109556 & A & 2720.349 & $-14.77 \pm 0.12$ & 0.47 & 13.74 & 7.4 & 0.13 & -5.20 & 10.04 & SB1? \\
\hline 61465 & & $\mathrm{~B}$ & 2720.368 & $4.78 \pm 0.16$ & 0.54 & 12.60 & 6.6 & 0.17 & -4.92 & 9.62 & SB1? \\
\hline 62596 & 111484 & A & 2720.301 & $-20.85 \pm 0.11$ & 0.55 & 12.57 & 6.6 & 0.21 & -4.73 & 9.32 & \\
\hline 62596 & & $\mathrm{~B}$ & 2720.317 & $-19.44 \pm 0.11$ & 0.56 & 11.91 & 6.2 & 0.18 & -4.84 & 9.50 & \\
\hline 63409 & 112747 & A & 2722.187 & $-3.65 \pm 0.12$ & 0.44 & 13.02 & 6.8 & 0.27 & -4.52 & 8.99 & \\
\hline 63403 & 112746 & $\mathrm{~B}$ & 2722.203 & $-4.01 \pm 0.57$ & 0.42 & & 45.5 & $>0.23$ & $>-4.63$ & $<9.17$ & \\
\hline 64030 & 113984 & $\mathrm{~A}$ & 2721.162 & $-91.81 \pm 0.13$ & 0.44 & 10.22 & 4.5 & 0.14 & -5.06 & 9.84 & SB1 \\
\hline 64030 & & B & 2721.172 & $-89.64 \pm 0.12$ & 0.54 & 8.49 & 3.0 & 0.14 & -5.12 & 9.92 & \\
\hline 65176 & 115863 & A & 2721.272 & $16.27 \pm 0.11$ & 0.54 & 10.72 & 5.2 & 0.15 & -5.05 & 9.82 & \\
\hline 65176 & & B & 2721.285 & $15.20 \pm 0.11$ & 0.69 & 7.62 & 2.5 & 0.18 & -4.90 & 9.58 & \\
\hline
\end{tabular}


S. Desidera et al.: Spectroscopic characterization of a sample of southern visual binaries, Online Material p 4

Table 4. continued.

\begin{tabular}{|c|c|c|c|c|c|c|c|c|c|c|c|}
\hline $\begin{array}{l}\text { Name } \\
\text { HIP }\end{array}$ & $\begin{array}{l}\text { Name } \\
\text { HD }\end{array}$ & Comp. & $\begin{array}{c}\text { JD } \\
-2450000\end{array}$ & $\begin{array}{c}R V \\
\mathrm{~km} \mathrm{~s}^{-1}\end{array}$ & $B-V$ & $\begin{array}{c}F W H M \\
\mathrm{~km} \mathrm{~s}^{-1}\end{array}$ & $\begin{array}{l}v \sin i \\
\mathrm{~km} \mathrm{~s}^{-1}\end{array}$ & $S$ & $\log R_{\mathrm{HK}}^{\prime}$ & $\begin{array}{c}\log \text { age } \\
y r\end{array}$ & Remarks \\
\hline 65352 & 116442 & $\mathrm{~A}$ & 2721.184 & $28.40 \pm 0.11$ & 0.77 & 6.22 & & 0.17 & -4.97 & 9.71 & \\
\hline 65355 & 116443 & B & 2721.193 & $27.44 \pm 0.12$ & 0.87 & 6.19 & 1.1 & 0.23 & -4.84 & 9.49 & \\
\hline 66874 & 119191 & A & 2497.980 & $-3.17 \pm 0.19$ & 0.42 & 35.82 & 20.7 & 0.17 & -4.88 & 9.55 & \\
\hline 66874 & & B & 2497.991 & $-4.01 \pm 0.16$ & 0.44 & 29.05 & 17.0 & 0.16 & -4.94 & 9.66 & \\
\hline 69328 & 124054 & A & 2720.268 & $-14.31 \pm 0.11$ & 0.58 & 7.57 & 1.9 & 0.16 & -4.97 & 9.71 & \\
\hline 69328 & & $\mathrm{~B}$ & 2720.280 & $-13.23 \pm 0.11$ & 0.64 & 7.10 & 1.4 & 0.16 & -5.02 & 9.77 & \\
\hline 70264 & 125628 & A & 2722.247 & $19.19 \pm 0.13$ & 0.86 & 10.40 & 5.5 & 0.21 & -4.88 & 9.56 & Giant, $\Delta V>1$ \\
\hline 70264 & & B & 2722.250 & $16.14 \pm 0.13$ & 0.45 & 12.28 & 6.3 & 0.18 & -4.80 & 9.43 & \\
\hline 70269 & 125906 & A & 2498.015 & $-33.39 \pm 0.11$ & & 8.04 & & 0.15 & & & \\
\hline 70269 & & B & 2498.030 & $-32.73 \pm 0.11$ & 0.56 & 8.58 & 3.2 & 0.14 & -5.12 & 9.92 & \\
\hline 70386 & 126246 & A & 2720.334 & $0.68 \pm 0.12$ & 0.54 & 13.65 & 7.4 & 0.33 & -4.40 & 8.61 & \\
\hline 70386 & & $\mathrm{~B}$ & 2720.343 & $1.66 \pm 0.11$ & 0.60 & 8.95 & 3.7 & 0.29 & -4.51 & 8.96 & \\
\hline 73674 & & A & 2498.060 & $-16.21 \pm 0.11$ & 0.61 & 8.05 & 2.8 & 0.14 & -5.09 & 9.89 & \\
\hline 73674 & 133131 & $\mathrm{~B}$ & 2498.969 & $-15.75 \pm 0.11$ & 0.61 & 7.88 & 2.5 & 0.15 & -5.05 & 9.82 & \\
\hline 74016 & 134066 & A & 2500.009 & $-33.57 \pm 0.29$ & 0.64 & & & & & & very low $\mathrm{S} / \mathrm{N}$ \\
\hline 74016 & & $\mathrm{~B}$ & 2499.996 & $-35.59 \pm 0.11$ & & & & 0.14 & & & \\
\hline 74432 & 135101 & A & 2722.226 & $-38.86 \pm 0.11$ & 0.69 & 7.44 & 2.3 & 0.16 & -4.99 & 9.74 & \\
\hline 74434 & & B & 2722.233 & $-38.76 \pm 0.11$ & 0.75 & 6.60 & 1.1 & 0.15 & -5.07 & 9.85 & \\
\hline 76446 & 138846 & $\mathrm{~A}$ & 2722.259 & $-69.47 \pm 0.11$ & 0.52 & 7.41 & 1.2 & 0.16 & -4.97 & 9.70 & $\Delta V>1$ \\
\hline 76446 & & B & 2722.276 & $-69.93 \pm 0.11$ & 0.76 & 6.71 & 1.4 & 0.22 & -4.80 & 9.43 & \\
\hline 76603 & 139461 & A & 2498.996 & $2.17 \pm 0.11$ & 0.48 & 10.41 & 4.8 & 0.15 & -5.01 & 9.76 & SB1 \\
\hline 76602 & 139460 & B & 2499.009 & $1.35 \pm 0.11$ & 0.52 & 12.90 & 6.8 & 0.17 & -4.92 & 9.62 & \\
\hline 76816 & 139864 & $\mathrm{~A}$ & 2721.327 & $-47.18 \pm 0.20$ & 0.54 & 25.05 & 14.7 & 0.21 & -4.71 & 9.29 & SB2 \\
\hline 76816 & & $\mathrm{~B}$ & 2721.361 & $-37.52 \pm 0.12$ & 0.93 & 6.95 & 2.5 & 0.26 & -4.83 & 9.48 & \\
\hline 78024 & 142661 & A & 2722.308 & $-37.87 \pm 0.11$ & 0.55 & 9.70 & 4.3 & 0.16 & -4.94 & 9.65 & $\Delta V>1$ \\
\hline 78024 & & B & 2722.315 & $-39.80 \pm 0.11$ & 0.82 & 7.08 & 2.3 & 0.43 & -4.46 & 8.84 & \\
\hline 78416 & 143215 & A & 2499.025 & $31.26 \pm 0.59$ & 0.55 & 55.99 & 30.7 & 0.27 & -4.52 & 8.99 & SB2 \\
\hline 78416 & & B & 2499.062 & $0.51 \pm 0.28$ & 0.54 & 71.91 & 37.4 & 0.36 & -4.35 & 8.23 & \\
\hline 79818 & 146368 & A & 2500.035 & $-25.21 \pm 0.12$ & 0.48 & 9.93 & 4.3 & 0.14 & -5.11 & 9.91 & SB1? \\
\hline 79818 & & $\mathrm{~B}$ & 2500.079 & $-20.20 \pm 0.12$ & 0.58 & 8.90 & 3.6 & 0.14 & -5.10 & 9.89 & SB1? \\
\hline 80399 & 147723 & A & 2499.969 & $-2.83 \pm 0.11$ & 0.59 & 10.07 & 4.7 & 0.13 & -5.25 & 10.10 & \\
\hline 80399 & 147722 & B & 2499.974 & $-1.27 \pm 0.11$ & & 8.95 & & 0.14 & & & \\
\hline 81394 & 149261 & A & 2498.092 & $-15.33 \pm 0.10$ & 0.51 & & & 0.20 & -4.75 & 9.35 & \\
\hline 81395 & 149262 & $\mathrm{~B}$ & 2499.103 & $-16.14 \pm 0.26$ & 0.49 & & & 0.15 & -5.00 & 9.74 & SB2 \\
\hline 84405 & 155885 & A & 2499.136 & $0.46 \pm 0.13$ & 0.86 & 7.55 & 3.0 & 0.40 & -4.54 & 9.02 & \\
\hline 84405 & 155886 & $\mathrm{~B}$ & 2499.141 & $-0.01 \pm 0.13$ & 0.86 & 7.40 & 2.8 & 0.38 & -4.56 & 9.06 & \\
\hline 99803 & 191869 & A & 2499.149 & $-18.93 \pm 0.23$ & 0.48 & 58.24 & 31.7 & 0.27 & -4.52 & 8.99 & \\
\hline 99803 & & $\mathrm{~B}$ & 2499.166 & $-17.83 \pm 0.27$ & 0.50 & 53.24 & 29.4 & 0.29 & -4.48 & 8.89 & \\
\hline 100045 & 192724 & A & 2498.151 & $-9.49 \pm 0.11$ & 0.53 & 19.36 & 11.2 & 0.13 & -5.21 & 10.05 & SB1? \\
\hline 100045 & & B & 2498.167 & $-2.74 \pm 0.11$ & 0.48 & 10.32 & 4.7 & 0.13 & -5.28 & 10.12 & \\
\hline 101292 & 195284 & $\mathrm{~A}$ & 2499.232 & $-21.45 \pm 0.12$ & & 8.32 & & 0.30 & & & \\
\hline 101292 & & $\mathrm{~B}$ & 2499.209 & $-21.51 \pm 0.12$ & & 8.17 & & 0.27 & & & \\
\hline 103438 & 199065 & A & 2500.211 & $10.92 \pm 0.10$ & 0.60 & 10.46 & 5.1 & 0.24 & -4.63 & 9.16 & \\
\hline 103438 & & $\mathrm{~B}$ & 2500.256 & $8.95 \pm 0.13$ & 0.76 & 9.96 & 5.0 & 0.44 & -4.39 & 8.54 & \\
\hline 104687 & 201796 & A & 2498.263 & $-20.73 \pm 0.11$ & 0.64 & 9.43 & 4.2 & 0.35 & -4.41 & 8.65 & \\
\hline 104687 & & B & 2500.116 & $-20.70 \pm 0.11$ & 0.71 & 9.54 & 4.5 & 0.34 & -4.48 & 8.90 & \\
\hline 110832 & 212746 & A & 2499.273 & $12.79 \pm 0.16$ & 0.58 & 25.06 & 14.7 & 0.31 & -4.46 & 8.84 & SB1? \\
\hline 110832 & 212746 & B & 2499.321 & $22.08 \pm 0.11$ & 0.60 & 12.01 & 6.3 & 0.13 & -5.26 & 10.11 & SB1? \\
\hline 114167 & 218269 & $\mathrm{~A}$ & 2498.322 & $3.88 \pm 0.13$ & 0.45 & 22.17 & 12.9 & 0.19 & -4.79 & 9.41 & \\
\hline 114167 & & B & 2498.330 & $4.60 \pm 0.12$ & 0.53 & 13.40 & 7.2 & 0.15 & -5.00 & 9.74 & low S/N \\
\hline 114914 & 219542 & A & 2498.191 & $-12.10 \pm 0.10$ & 0.64 & 8.13 & 2.9 & 0.15 & -5.07 & 9.85 & \\
\hline 114914 & & B & 2498.209 & $-10.99 \pm 0.12$ & 0.71 & 7.86 & 2.9 & 0.20 & -4.81 & 9.45 & \\
\hline & 38392 & & 2719.988 & $-9.57 \pm 0.13$ & 0.94 & 7.12 & 2.8 & 0.50 & -4.53 & 9.00 & HK standard \\
\hline & 38393 & & 2719.978 & $-9.21 \pm 0.12$ & 0.48 & 14.02 & 7.6 & 0.14 & -5.10 & 9.90 & HK standard \\
\hline & 38393 & & 2721.990 & $-9.22 \pm 0.12$ & 0.48 & 14.24 & 7.8 & 0.14 & -5.09 & 9.88 & HK standard \\
\hline & 45067 & & 2721.980 & $47.25 \pm 0.11$ & 0.56 & 10.97 & 5.4 & 0.13 & -5.17 & 10.00 & HK standard \\
\hline & 76151 & & 2721.985 & $31.91 \pm 0.10$ & 0.67 & 7.14 & 1.7 & 0.22 & -4.73 & 9.33 & HK standard \\
\hline & 115617 & & 2721.150 & $-7.82 \pm 0.10$ & 0.71 & 7.15 & 1.9 & 0.16 & -5.03 & 9.79 & HK standard \\
\hline & 152391 & & 2721.302 & $45.13 \pm 0.10$ & 0.76 & 8.28 & 3.5 & 0.42 & -4.42 & 8.67 & HK standard \\
\hline
\end{tabular}

